Research Article

\title{
An Improved Backward/Forward Sweep Power Flow Method Based on a New Network Information Organization for Radial Distribution Systems
}

\author{
Saad Ouali $(\mathbb{D}$ and Abdeljabbar Cherkaoui \\ Laboratory of Innovative Technologies (LTI), National School of Applied Sciences, Abdelmalek Essaâdi University, Tangier, \\ Morocco \\ Correspondence should be addressed to Saad Ouali; saad.ouali1@gmail.com
}

Received 17 September 2019; Revised 29 October 2019; Accepted 26 December 2019; Published 17 January 2020

Academic Editor: Salvatore Favuzza

Copyright ( $) 2020$ Saad Ouali and Abdeljabbar Cherkaoui. This is an open access article distributed under the Creative Commons Attribution License, which permits unrestricted use, distribution, and reproduction in any medium, provided the original work is properly cited.

\begin{abstract}
This paper presents a load flow algorithm based on the backward/forward sweep principle, flexible with network topology changes, with an improvement in ensuring a minimum number of searching for connections between nodes in the calculation sequence in the forward and the backward sweep, by organizing the radial distribution system information into a main line and its derivations. The proposed load flow analysis is easy to implement and does not require the use of any complex renumbering of branches and nodes, or any matrix calculation, with the only use of linear equations based on Kirchhoff's formulation.
\end{abstract}

\section{Introduction}

The load consumed or produced in medium- and lowvoltage distribution network at each bus can be measured by electronic meters [1] and can be transmitted through advanced metering infrastructures [2], or it can be estimated from the forecast of load demand [3] and the solar [4] or wind prevision [5]. However, knowing the loads at each network node is not sufficient to conceive network control applications, and further information is needed, such as voltage magnitude and voltage deviation of each network node; capturing that information directly requires serious investments in sensors and new communication infrastructure [6]. Avoiding those investments is possible, thanks to a tool named "load flow analysis" [7], which is able to calculate the needed network information only from the values of load consumed or injected of each node. Load flow analysis is a widely used tool transmission system in several applications, as power generating scheduling [8], and could also be very useful for many applications in distribution networks, such as network analysis [9], load control [10, 11], network reconfiguration [12], integration of generation [13], optimal allocation of UPQC [14], and integration of electric vehicle [15]. Several methods had been developed and deployed in the transmission system such as Gauss-Seidel [16], Newton-Raphson [17] and fast-decoupled methods [18].

Unlike transmission systems, the conductors used in distribution networks are characterized by high $R / X$ ratio [19] and a radial structure with large number of nodes, branches, distributed generation [20], and complex topology configurations [21], which can be changed for maintenance activities, emergency operations, or network configurations. Due to the high $R / X$ ratios, transmission grid load flow methods, or the Jacobian-based methods, such as Newton-Raphson, Gauss-Seidel, and fast-decoupled methods, failed with such networks $[22,23]$; even though with some advancements in the Newton-Raphson methods, the robustness of the program is obtained, but still the computational time is large [24, 25]. As the Gauss implicit Z-matrix presented in [26], adapted for load flow analysis of radial and weakly meshed electrical systems, the time computation required for solving a set of equations whose number is proportional to the network nodes is large. 
The restrictions of the Jacobian-based methods in dealing with distribution systems have been revealed in a number of papers, where the authors complained that the transmission grid load flow methods were not appropriate to solve practical problems presented when analyzing distribution systems $[27,28]$ and prefer backward/forward sweepbased approach. A powerful mathematical efforts had been developed in [29], proving the convergence of the backward/ forward algorithms. Also the existence of a unique solution of the load flow in distribution networks had been proved in [30].

The backward/forward sweep approach had been developed and presented in [31]; however, the authors do not define how to automatically identify the end of each branch, and from where to start the backward calculation, for a program code preparation. A direct method of backward/ forward sweep is presented in [32], and based on the development of two matrices from the topological structure of the network: bus injection to bus current (BIBC) and bus current to bus voltage (BCBV), the solution is obtained by a simple matrix multiplication of BIBC and BCBV. However, for an electrical system with $m$ branches and $n$ buses, the dimension of the BIBC is " $m x(n-1)$," and the size of BCBV is " $(n-1) x m$." In medium-voltage networks, the number of branches and buses is very important, and every MV/LV substation, switching substations, or a network derivation represent a bus. In low-voltage networks, the number of branches and buses is even more important as every connector forms a network deviation or consumer connection, and also every electric LV switchboard represents a bus. The BIBC and BCBV are used in every iteration of the algorithm which may impact the time-consumption of computation and make the method not fast enough for real-time applications.

To avoid the use of matrix calculations with large dimension matrices in every iteration of the backward and the forward sweep process, others methods had been developed. In [33], a feeder-lateral-based method was adopted which requires the division of network buses and branches into several layer, and then the network topology information was converted to a "layer-lateral-based data format"; however, the definition of layers is very complex and can risk confusion in the development of the program code. In [22], authors had developed a method based on first converting the network topology information into a defined format and starting a renumbering process of nodes and branches, after organizing the network information into a defined format, and they obtained a numeration of network branches and buses. Each network element was defined by four information: "node behind," "line-ahead-1," "line-ahead-2," and "terminal node," and then, an iterative process was started by first ignoring the effect of transmission losses, and assuming that the total power crossing a node was accumulated at the node itself, the voltage of the node was calculated knowing the voltage of the node behind. The line losses are terminated, the actualization of the total power calculated is performed, and the process was continued till the difference between the calculated power losses from two iterations was within tolerance limits. However, the development of this method is made supposing that the intermediate nodes are having one incoming branch and at most two outgoing branches, which is not the true in real networks, as the case of networks with switching substations. This supposing makes this method not effective or leads to conversion of the intermediate nodes with more than two outgoing nodes to several nodes with at most two outgoing nodes and many virtual branches, which could increase the number of nodes and branches. Also all the previous methods presented are not flexible with changes in network topology and are developed assuming that the topology configuration of distribution systems is fix.

In this paper, we contribute a new approach of the load flow analysis with a method that does not require the use of any complex renumbering of nodes and branches, or any matrix calculation, flexible with network topology modification. The proposed method is an iterative procedure based on the backward/forward sweep algorithm with the only use of Kirchhoff's formulation. From a dynamic data of system, a preliminary calculation is made to organize the radial distribution information into a main line and its derivations. After the organization of the system data information into a suitable model, voltages of all other nodes are set to the nominal voltage, an iterative process is started by calculating currents in derivation lines, and then the branch current in the main line in a backward sweep is computed using Kirchhoff's current law. The node voltages are updated in a forward sweep using the voltage drop calculation, and this backward and forward sweep is repeated until voltage magnitudes at each node in the present iteration and the previous iteration are lower than a tolerance limit. The convergence of the proposed algorithm is guaranteed due to the use of the backward/forward sweep method in the iterative process, and the only difference made in the organization of the distribution information is to facilitate the programming by ensuring a minimum number of searching for connections between nodes. A unique IEEE 15-bus feeder, two meshed IEEE 15-bus feeders, and also an IEEE 69-bus had served as a case study for performing the test of the proposed method, and the results obtained are compared with three other existing load flow methods presented in [34]: primitive impedance-based distribution load flow (PIDLF), current injection-based distribution load flow (CIM), and fast-decoupled single matrix model distribution load flow (SMM).

The strength of the approach presented in this paper lies on its simplicity to be implemented, where the complex branch renumbering is not needed and it is independent from the first numbering of nodes. The reduction of flow calculation is obtained by ensuring a minimum number of searching for connections between nodes and by using only simple algebraic expressions without any trigonometric functions or matrix calculation. The proposed load flow method is valid for radial distribution system with or without distributed generations, and it is flexible for the network topology reconfiguration.

In what follows, we present the problem formulation and what the load flow is done for in Section 2. Next in Section 3, we provide the theoretical foundation and the proposed 
method. In Section 4, a detailed simulation is done with a small network of 15 buses to more understand the presented method and how to identify the main line and derivation lines, and a comparison of the results obtained is done with other existing load flow methods. Section 5 concludes this paper.

\section{Load Flow Analysis}

Load flow analysis requires two inputs to provide electrical network characteristics. These two inputs are line data and load data.

Load data can be established by collecting the active power injections and the consumptions of the active/reactive powers at each node. That network information can be assembled in one table, as shown in Table 1.

Load data can be updated either by exploring digital meters information, or by forecasting load demand. In Morocco, digital meters are available and had been already installed and explored at several MV and HV consumers. Advanced Metering Infrastructure (AMI) could concentrate digital meters information and could provide utility companies with real-time load data about power production and/or consumption.

Line data represent network conductor characteristics; in utilities, line data are grouped in the geographic system information (GIS), or it can be brought together manually by knowing the electrical characteristics of conductors used, the laying mode, and the year of the conductor laying. The network information can be assembled in one table, as shown in Table 2.

However, the topology structure of the network can be changed for maintenance activities, emergency operations, or network configurations.

Medium-voltage network SCADA, already existing in $\mathrm{HV} / \mathrm{MV}$ substations in Morocco, is used as the MV network remote control system to handle network operations and to receive $\mathrm{MV} / \mathrm{LV}$ substation details. The SCADA can remote monitor the MV switch status of the MV/LV substations. So any change in the topology structure can be detected by this SCADA, and this information can be used to update the line data table. In low-voltage distribution systems, the changes in topology can be done by manual entries as the topology reconfiguration in $\mathrm{LV}$ systems is done manually by the distribution network technician.

The diagram of the general principle of the proposed load flow method is given in Figure 1.

\section{Proposed Load Flow Analysis Method}

The backward/forward sweep approach is based on three key steps [35].

Starting from the terminal node, considering the electrical characteristics presented in Figure 2 the following three key steps are formulated.

Step 1 (nodal current calculation): the current injection at each node " $i$ " is calculated using the following equation:
TABLE 1: Load data table.

\begin{tabular}{lcc}
\hline Node & kVA & kVAr \\
\hline$\ldots$ & $\ldots$ & $\ldots$ \\
$\ldots$ & $\ldots$ & $\ldots$ \\
$\ldots$ & $\ldots$ & $\ldots$ \\
\hline
\end{tabular}

TABLE 2: Line data table.

\begin{tabular}{lccc}
\hline Sending node & Receiving node & $R(\mathrm{Ohm})$ & $X(\mathrm{Ohm})$ \\
\hline$\ldots$ & $\ldots$ & $\ldots$ & $\ldots$ \\
$\ldots$ & $\ldots$ & $\ldots$ & $\ldots$ \\
$\ldots$ & $\ldots$ & $\ldots$ & $\ldots$ \\
\hline
\end{tabular}

$$
I_{i}^{(k)}=\operatorname{conj}\left(\frac{P_{n-i}+j * Q_{n-i}}{V_{n-i}^{k}}\right), \quad i=1,2, \ldots, n,
$$

where $S_{i}$ is the power injection at node $i$ and $V(k) I$ is the voltage of node $i$ calculated from iteration $k$.

Step 2 (backward sweep): starting from the last ordered branch, current $J i, i+1$, in branch from the node $i$ to the node " $i+1$ " is calculated using the following equation:

$$
J_{n-i}^{k}=-\operatorname{conj}\left(\frac{P_{n-i}+j * Q_{n-i}}{V_{n-i}^{k}}\right)+\sum_{r} J_{n-r}^{k}, \quad r=1, \ldots,
$$

where $\sum_{r} J_{n-r}^{k}$ is the current in branches emanating from node " $i$."

Step 3 (forward sweep): starting from the root bus, the node voltages are updated using the following equation:

$$
V_{i}^{(k)}=V_{i-1}^{(k)}-Z_{i} J_{i}^{(k)}, \quad i=2,3, \ldots, n,
$$

where $Z_{i}$ is the series impedance of branch " $i-1$, $i$."

Those three steps are repeated until voltage magnitudes at each node in the present iteration and the previous iteration is lower than a tolerance limit $\varepsilon$ :

$$
\max \left(\left[V^{(k+1)}\right]-\left[V^{(k)}\right]\right)<\varepsilon .
$$

However, it is needed to highlight that, in distribution systems, there are three kinds of buses: terminal node, common-intermediate node, and simple-intermediate node, as shown in Figure 3.

If a network had no common buses and no derivation lines outgoing from those common buses, the branch current calculated in the forward sweep will be calculated only by the use of equation (2). The presence of common buses and derivations had lead to develop several methods to define landmarks able to help browsing all network branches. As used in reference [9], network elements are divided into several layers with no common buses which lead to the use of equation (2) in each layer and then the results of each layer are added to get the current at the bus of the substation. In [8], the authors in order to avoid browsing network elements and searching from where to start and which path to follow in the backward and the forward sweep, 


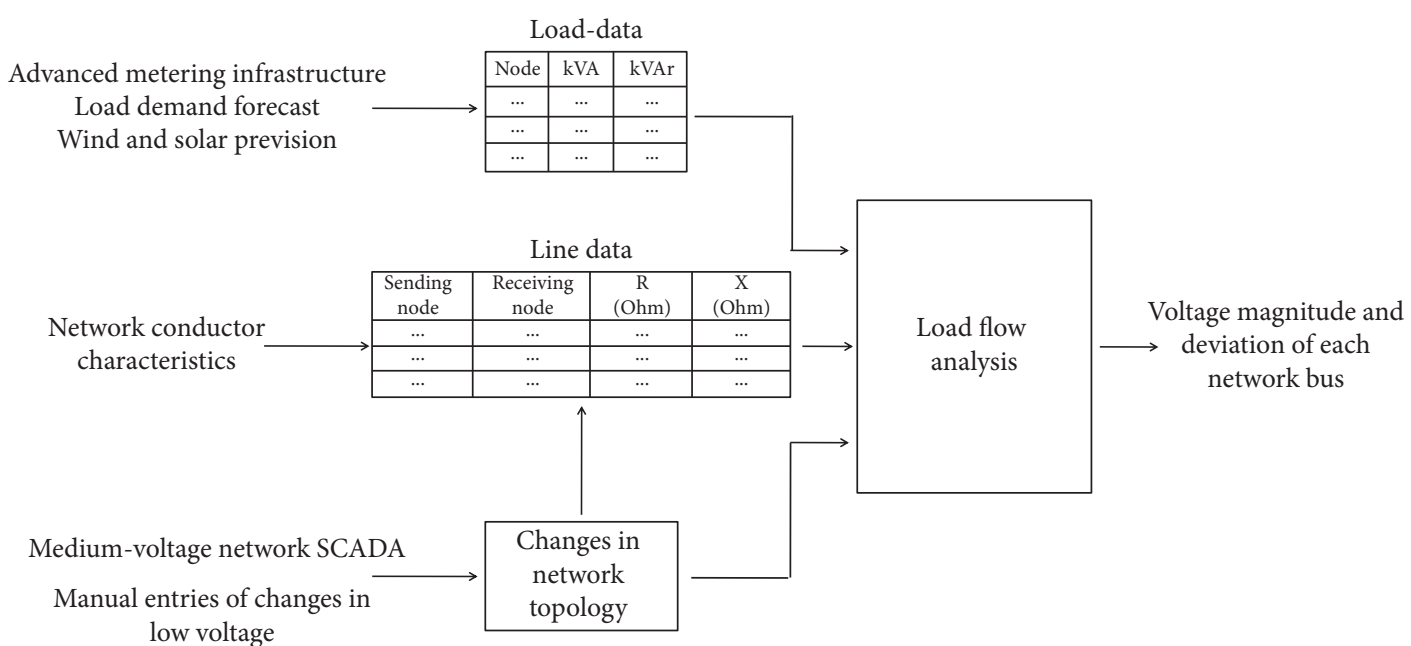

Figure 1: Diagram of the proposed load flow analysis.

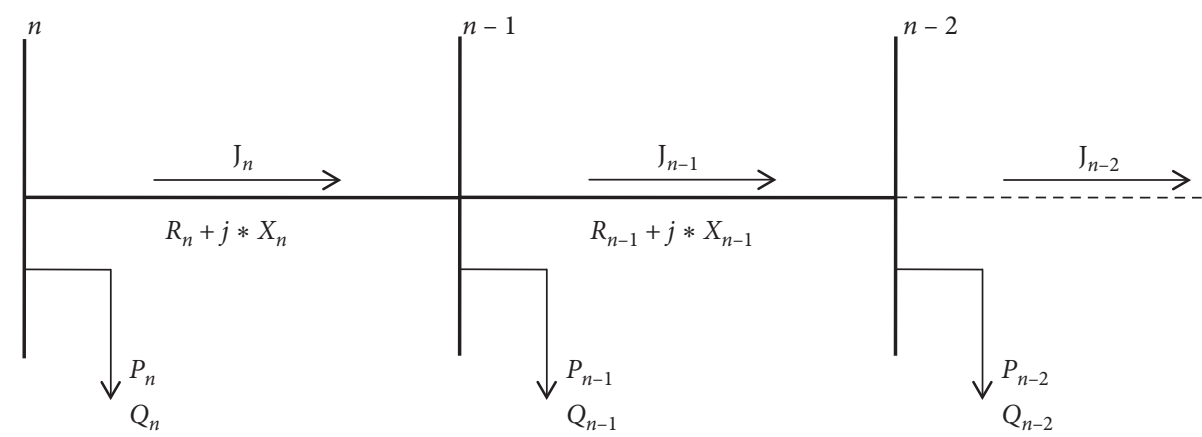

Figure 2: Single line diagram.

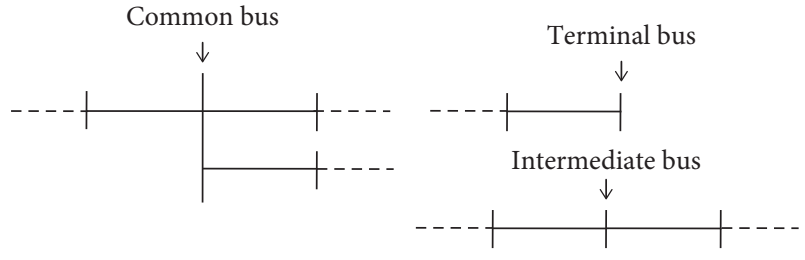

Figure 3: Types of network buses.

they use two matrices, BIBC and BCBV. However, the use of the matrices is repeated in each iteration, which impact the time computation of the method.

In this paper, a novel method is presented based on the idea of a new element ordering by dividing the network elements into a main line and its derivations and the use of the matrix $D$ as a landmark to browse all network elements. The proposed element ordering process is carried out to determine the calculation sequence in the forward and the backward sweep.

So, from a dynamic line data of the system, a preliminary calculation is made to identify the main line and its common buses. The main line, in this paper, is chosen as the longest sequence of nodes.

It is needed to highlight that the main line can be chosen according to the need: the most loaded line or the line allowing a backup in the case of the open loop structure. The derivation lines are the lines outgoing from the common buses of the main line.

To facilitate the development of the program code, derivation lines outgoing from the common buses of the main line are organized into a matrix named " $D$," and a presentation of the matrix is shown in Figure 4.

$\mathrm{C} 1$ the first common node of the main line and $\mathrm{C} 2$ the second common node, and $\mathrm{CN}$ are the common bus in the derivation lines.

The matrix $D$ is developed by putting the first common bus of the main line and listed its elements. Each time a bus is spotted as a common bus, a new column is added with the new subderivations till getting a terminal bus. The number of column of the first common bus is saved, and the process continues until all common buses of the main line are listed.

After the organization of the line data information, the next step is to read the load data, convert it into a per-unit value, and set the voltages of all nodes to the nominal voltage "1 p.u."

By preparing the line data and the load data information into a suitable model, an iterative procedure based on backward/forward sweep algorithms is started.

3.1. The Backward Sweep. This step is based on the fact that the complex power, measured by electronics meters and estimated by load demand forecast, is known at each node. 


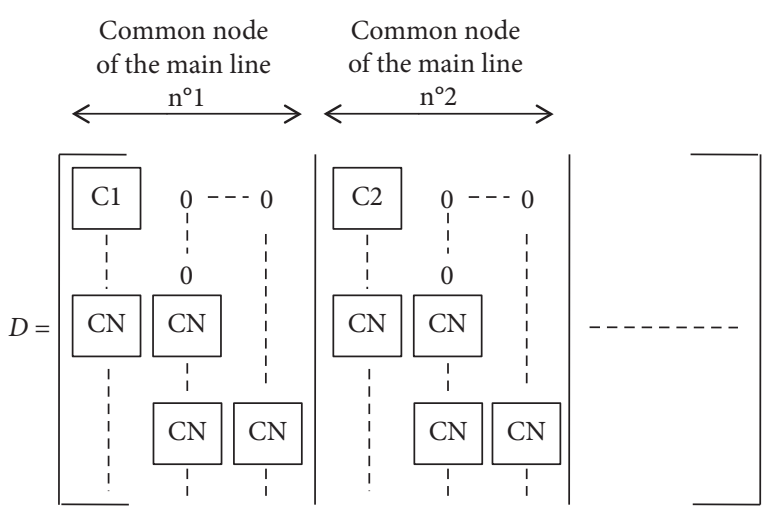

FIgURE 4: Derivation matrix.

The first step in the backward step is calculating branch currents in derivation lines and then computing the branch current on the main line in a backward sweep using Kirchhoff's current law.

Starting from the last column of each common bus in the matrix $D$, the current is calculated using equation (2) and put at a new line created at the bottom of the matrix " $D$." Each time a common bus is spotted, the current between the common bus and the bus before is computed by the following equation:

$$
J_{n-i}^{k}=-\operatorname{conj}\left(\frac{P_{n-i}+j * Q_{n-i}}{V_{n-i}^{k}}\right)+\sum_{r} J_{n-r}^{k}+D i,
$$

where $D i$ is the element of matrix $D$ in the last line and the column number " $i-1$," which represents the sum of the branch current emanating from the common bus.

The next step in the backward sweep is the calculation of the branch current in every branch of the main line. For a simple-intermediate or terminal bus, the branch current is obtained using equation (2). If the bus of the main line is a common bus, the current in the next branch is obtained using equation (6).

The electric current between a sending node $(n-i)$ and a receiving node $(n-i-1)$ in the main line is

$$
J_{n-i}^{k}=-\operatorname{conj}\left(\frac{P_{n-i}+j * Q_{n-i}}{V_{n-i}^{k}}\right)+\sum_{r} J_{n-r}^{k}+\sum_{\text {derivation }} J_{i}^{0},
$$

where $\sum_{\text {derivation }} J_{i}^{0}$ is the current in branches emanating from the common bus of the main line.

3.2. The Forward Sweep. Using the computed current, an updating of voltage values starts from the root bus. The node voltages are updated using the equation (3).

This backward and forward sweep is repeated until voltage magnitudes at each node in the present iteration and the previous iteration are lower than a tolerance limit. The flow chart of the method is given in Figure 5.

\section{Simulation Results}

The proposed method program code is made in MATLBAB and tested on IEEE 15-bus distribution network, which have a

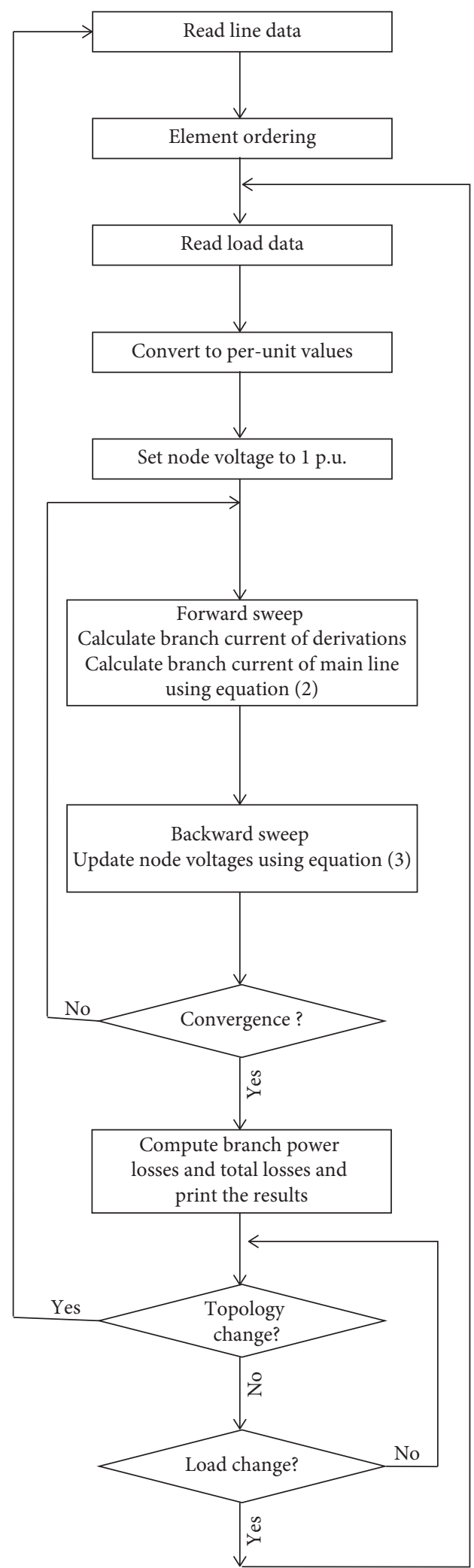

FIgURE 5: Flow chart of the proposed method.

similar structure as Moroccan medium-voltage distribution system. The line data are given in Table 3, and the load data are presented in Table 4. The power factor of the load is 
TABle 3: Line data of the 15-bus system.

\begin{tabular}{lccc}
\hline Sending node & Receiving node & $R(\mathrm{Ohm})$ & $X(\mathrm{Ohm})$ \\
\hline 1 & 2 & 1.530 & 1.778 \\
2 & 3 & 1.037 & 1.071 \\
3 & 4 & 1.224 & 1.428 \\
4 & 5 & 1.262 & 1.499 \\
2 & 9 & 1.176 & 1.335 \\
9 & 10 & 1.100 & 1.190 \\
2 & 6 & 1.174 & 1.332 \\
6 & 7 & 1.174 & 1.332 \\
6 & 8 & 1.174 & 1.323 \\
3 & 11 & 1.150 & 1.285 \\
11 & 12 & 1.274 & 1.522 \\
12 & 13 & 1.274 & 1.522 \\
4 & 14 & 1.075 & 1.522 \\
4 & 15 & 1.075 & 1.522 \\
\hline
\end{tabular}

TABLE 4: Load data of the 15-bus system.

\begin{tabular}{lc}
\hline Node & $\mathrm{kVA}$ \\
\hline 1 & 00.0 \\
2 & 63.0 \\
3 & 100.0 \\
4 & 200.0 \\
5 & 63.0 \\
6 & 200.0 \\
7 & 200.0 \\
8 & 100.0 \\
9 & 100.0 \\
10 & 63.0 \\
11 & 200.0 \\
12 & 20.0 \\
13 & 100.0 \\
14 & 100.0 \\
15 & 200.0 \\
\hline
\end{tabular}

assumed to be 0.7 [11]. The single-phase diagram of the test system is presented in Figure 6.

From the dynamic line data, presented in Table 1, a preliminary calculation is made to organize the radial distribution information into a suitable model: a "main line" and its "derivations."

The main line, the line containing the largest number of successive nodes, of the 15-bus system is [ $\left.\begin{array}{llllllll}1 & 2 & 3 & 11 & 12 & 13\end{array}\right]$, with 6 nodes (shown as red line in the Figure 11), and the three derivations have been identified from the common nodes 2 and 3 .

The matrix $D$ is shown in Figure 7.

The element ordering of the IEEE 15-bus is presented in Figure 8.

After the organization of the line data information into a main line and its derivations, the next step is to read the load data, presented in Table 2, convert it into a per-unit value, and set all the voltage value of all nodes to " 1 p.u."

Base voltage and base power are considered as $11 \mathrm{kV}$ and $100 \mathrm{kVA}$, respectively.

By preparing the line data and the load data information into a suitable model, an iterative procedure based on backward/forward sweep algorithms is started, and the convergent criterion is chosen as 0.0001 .

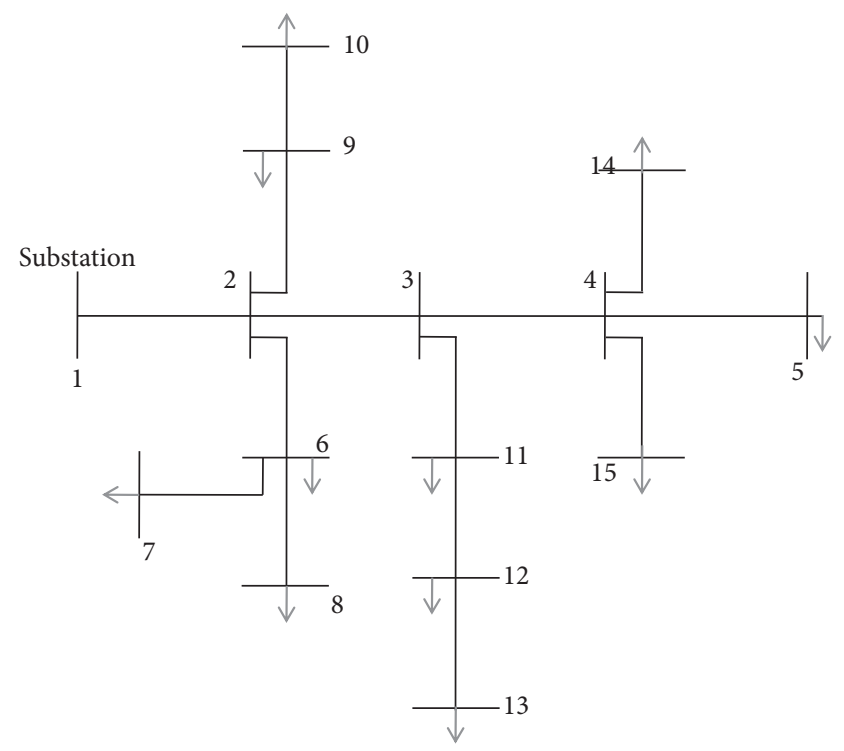

FIgURE 6: Single-phase diagram of the 15-bus distribution system.

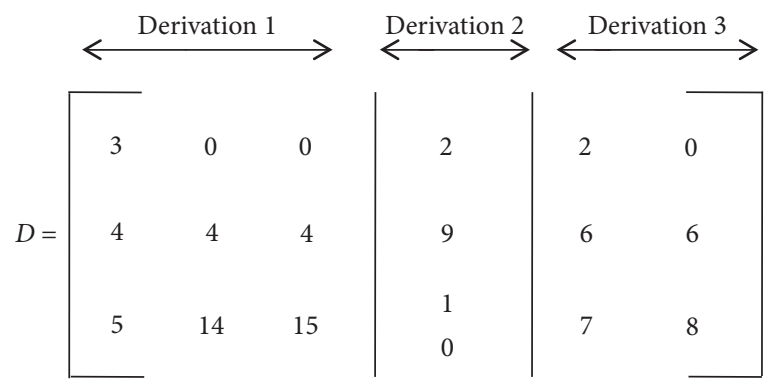

Figure 7: The matrix of derivations.

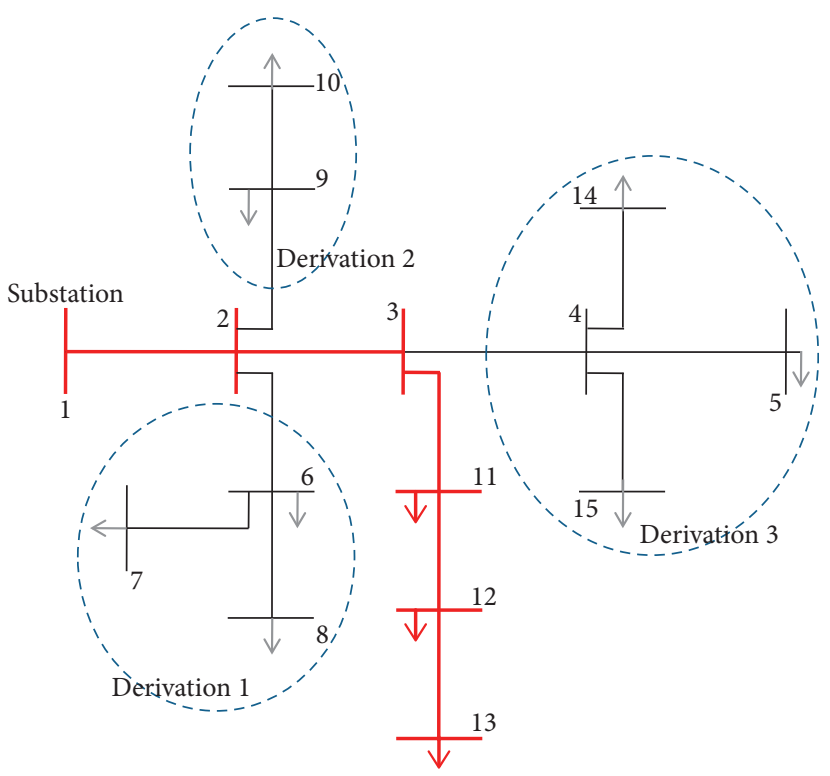

Figure 8: The main line and its derivation of the 15-bus system.

The results obtained were been compared with three other existing load flow methods [11]: primitive impedancebased distribution load flow (PIDLF), current injections- 
TABle 5: Results for IEEE 15-bus system.

\begin{tabular}{lcccc}
\hline Node & CIM & PIDLF & SMM & Proposed method \\
\hline 1 & 1 & 1 & 1 & 1 \\
2 & 0.97128 & 0.96885 & 0.97031 & 0.97017 \\
3 & 0.95667 & 0.95427 & 0.95571 & 0.95657 \\
4 & 0.9509 & 0.94852 & 0.94995 & 0.95078 \\
5 & 0.94991 & 0.94754 & 0.94896 & 0.94977 \\
6 & 0.95822 & 0.95583 & 0.95726 & 0.96267 \\
7 & 0.95476 & 0.95237 & 0.9538 & 0.95974 \\
8 & 0.95694 & 0.95455 & 0.95599 & 0.96115 \\
9 & 0.96797 & 0.96555 & 0.967 & 0.96020 \\
10 & 0.96689 & 0.96448 & 0.96593 & 0.95936 \\
11 & 0.94995 & 0.94757 & 0.949 & 0.95202 \\
12 & 0.94582 & 0.94346 & 0.94488 & 0.95006 \\
13 & 0.94451 & 0.94215 & 0.94357 & 0.94842 \\
14 & 0.9486 & 0.94623 & 0.94766 & 0.94926 \\
15 & 0.94844 & 0.94606 & 0.94749 & 0.94775 \\
\hline
\end{tabular}

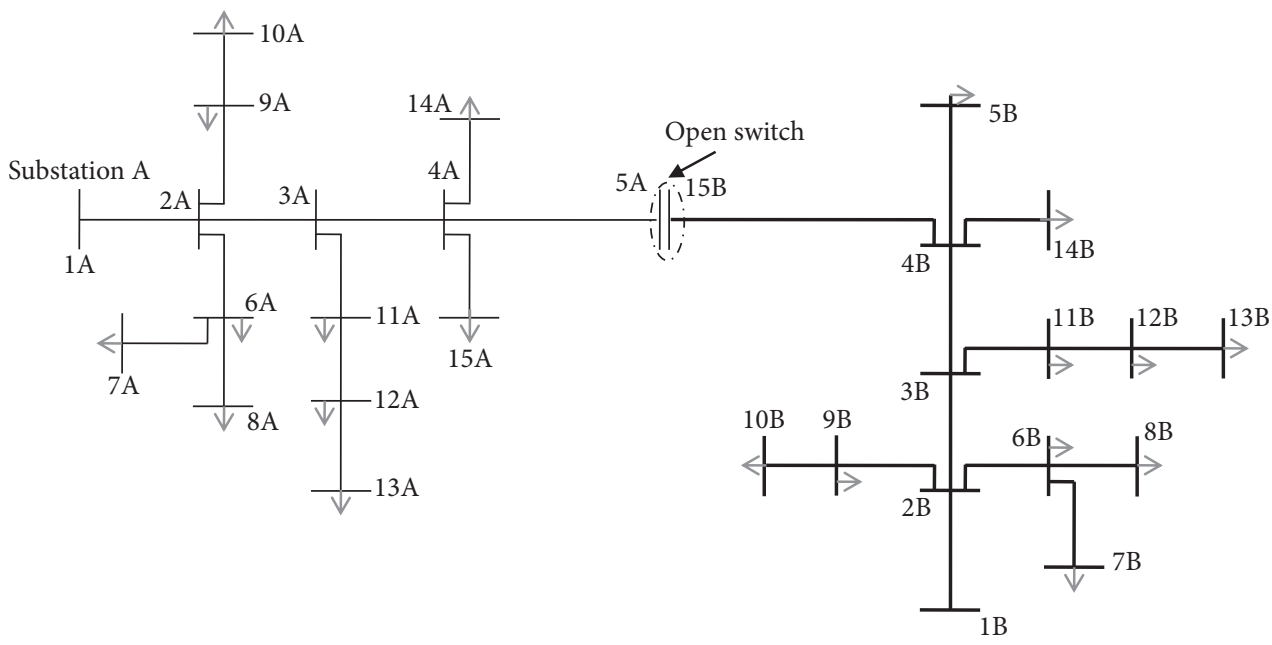

Substation B

FIGURE 9: Single-phase diagram of two meshed 15-bus distribution systems.

based distribution load flow (CIM), and fast-decoupled single matrix model distribution load flow (SMM). The results are summarized in Table 5 .

To simulate the flexibility of the proposed power flow method, let us consider that the IEEE 15-bus feeder used is meshed with another 15-bus feeder but from two different substations: Substation A and substation B, as shown in Figure 9.

A network topology may occur by changing the state of sectionalize switches and tie switches. Figure 10 presents a topology structure resulting from the previous scheme, by closing the node " $5 \mathrm{~A}-15 \mathrm{~B}$ " and opening the node $2 \mathrm{~A}$.

The new main line of the feeder supplied from substation $\mathrm{B}$ is $\{1 \mathrm{~B}, 2 \mathrm{~B}, 3 \mathrm{~B}, 4 \mathrm{~B}, 5 \mathrm{~A} / 15 \mathrm{~B}, 4 \mathrm{~A}, 3 \mathrm{~A}, 2 \mathrm{~A}, 9 \mathrm{~A}, 10\}$. The element ordering of the new feeder is presented in Figure 11.

By applying the proposed approach, the voltage magnitude obtained is reported in Figure 12.
To prove the effectiveness of the proposed with largest network, another application is done with the IEEE 69-bus system, and Figure 13 presents the single-phase diagram of the network.

Table 6 resumes the network characteristics and the results obtained by the proposed method and the results obtained by the three other existing methods: primitive impedance-based distribution load flow (PIDLF), current injection-based distribution load flow (CIM), and fastdecoupled single matrix model distribution load flow (SMM).

Comparison of the computational performance of the proposed approach with other methods is presented in Table 7.

From Table 6, we can observe that, for small system, the number of iteration is the same for the 4 methods; however, with bigger systems, the proposed approach and the SMM take less computational times compared to the two others methods. 


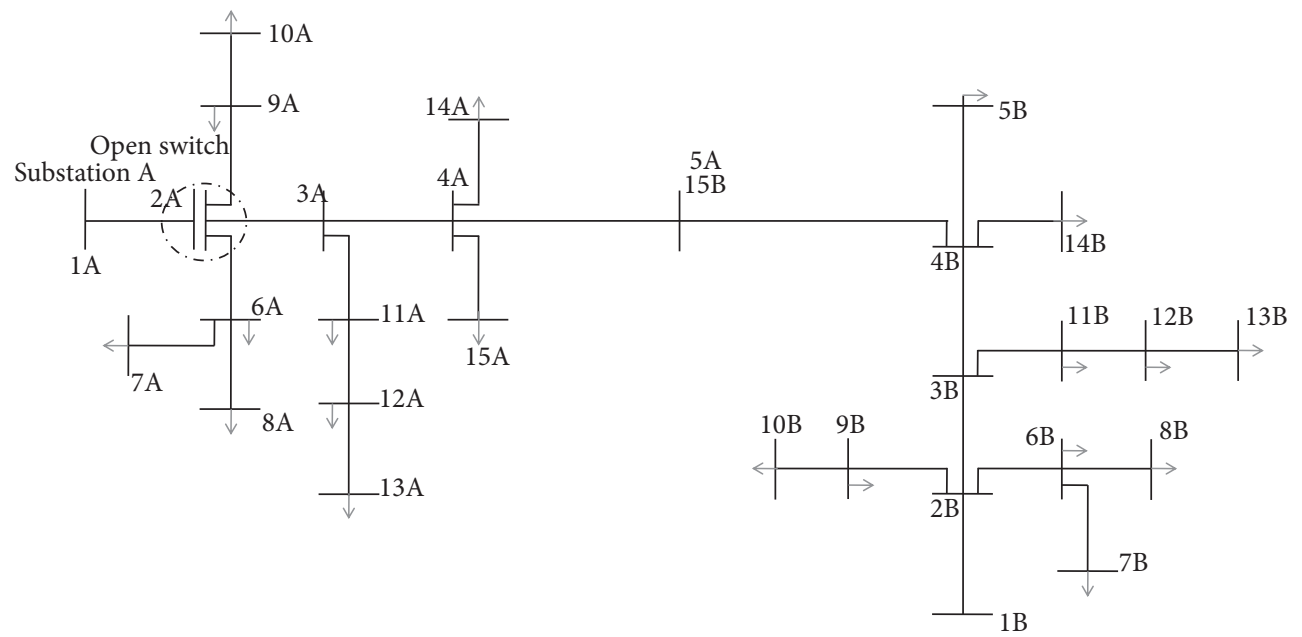

Substation B

Figure 10: Topology structure resulting from closing the node 5A-15 and opening the switch of the node $2 \mathrm{~A}$.

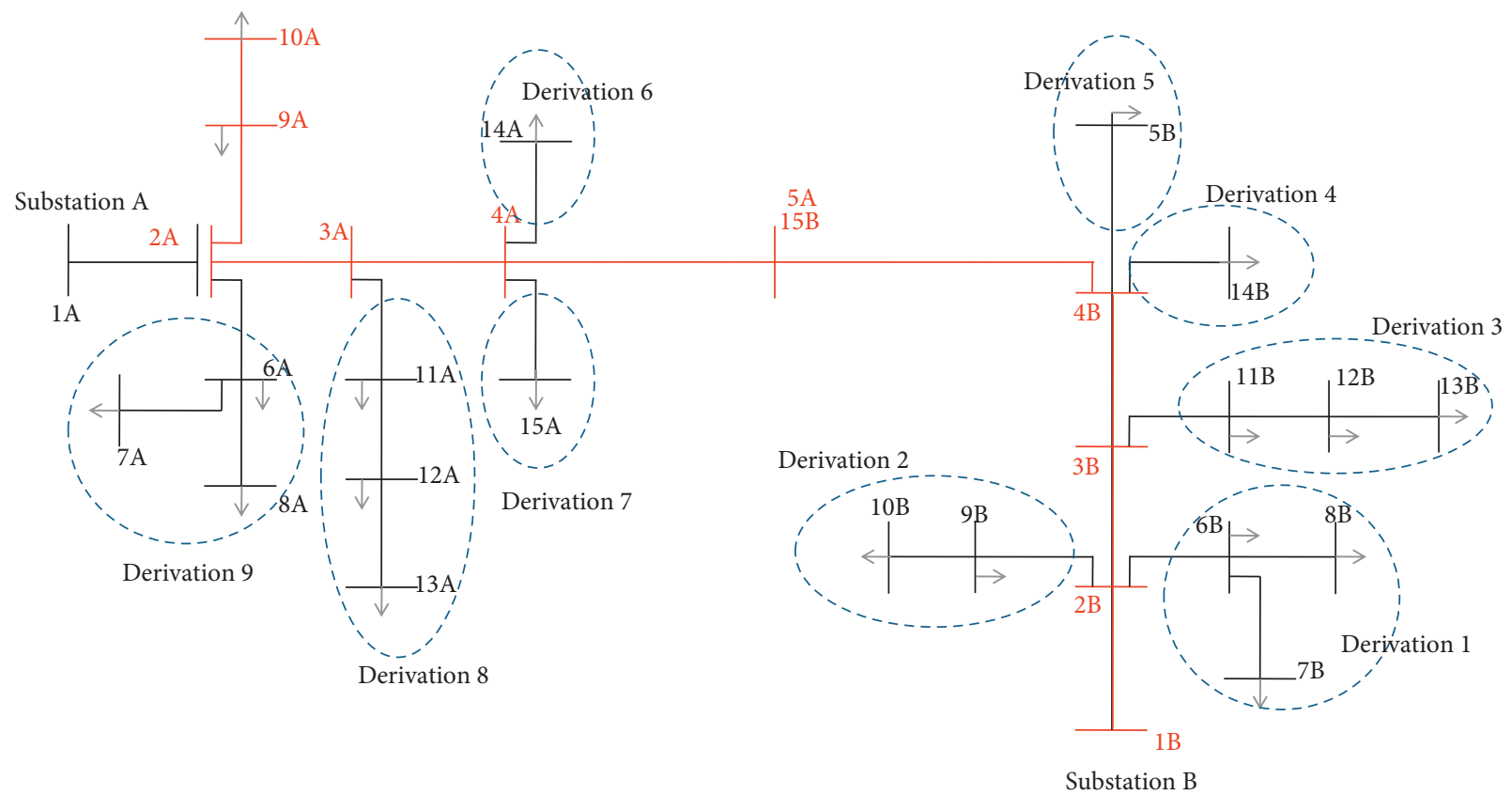

FIgURE 11: The element ordering of the topology structure presented in Figure 10.

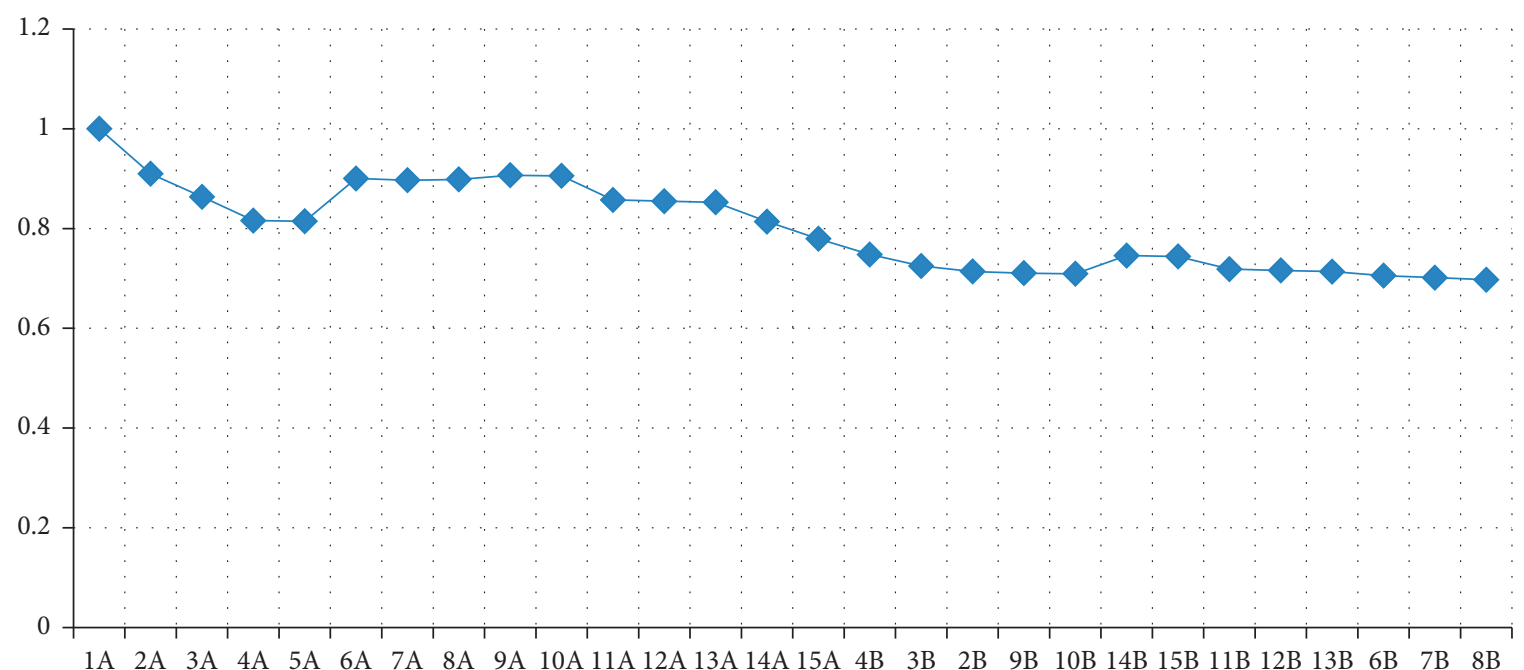

FIGURE 12: Voltage magnitudes of topology structure presented in Figure 10. 


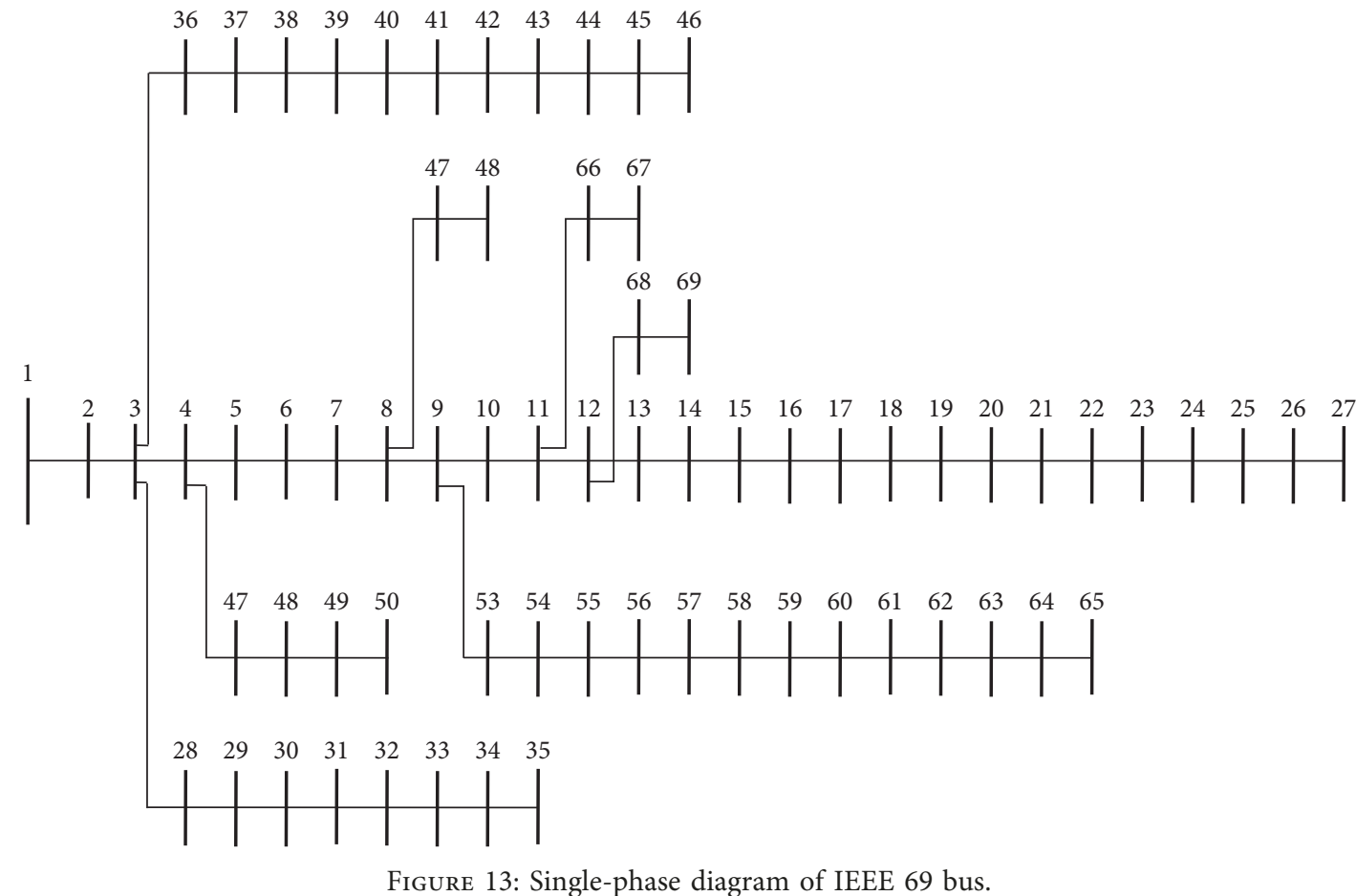

TABle 6: Results for IEEE 69-bus system.

\begin{tabular}{lcccc}
\hline Node & CIM & PIDLF & SMM & Proposed method \\
\hline 1 & 1 & 1 & 1 & 1 \\
2 & 0.999977 & 0.997477 & 0.998977 & 0.998890 \\
3 & 0.999953 & 0.997453 & 0.998953 & 0.998866 \\
4 & 0.999900 & 0.997400 & 0.998900 & 0.998813 \\
5 & 0.999330 & 0.996832 & 0.998331 & 0.998244 \\
6 & 0.993216 & 0.990733 & 0.992223 & 0.992137 \\
7 & 0.986994 & 0.984527 & 0.986007 & 0.985922 \\
8 & 0.985490 & 0.983026 & 0.984505 & 0.984419 \\
9 & 0.984708 & 0.982246 & 0.983723 & 0.983638 \\
10 & 0.976279 & 0.973838 & 0.975303 & 0.975218 \\
11 & 0.974354 & 0.971918 & 0.973380 & 0.973295 \\
12 & 0.967557 & 0.965138 & 0.966589 & 0.966506 \\
13 & 0.964086 & 0.961676 & 0.963122 & 0.963039 \\
14 & 0.960598 & 0.958197 & 0.959637 & 0.959554 \\
15 & 0.957287 & 0.954894 & 0.956330 & 0.956247 \\
16 & 0.956745 & 0.954353 & 0.955788 & 0.955706 \\
17 & 0.955774 & 0.953385 & 0.954818 & 0.954736 \\
18 & 0.955762 & 0.953373 & 0.954806 & 0.954724 \\
19 & 0.955192 & 0.952804 & 0.954237 & 0.954154 \\
20 & 0.954976 & 0.952589 & 0.954021 & 0.953939 \\
21 & 0.954645 & 0.952258 & 0.953690 & 0.953608 \\
22 & 0.954633 & 0.952246 & 0.953678 & 0.953596 \\
23 & 0.954496 & 0.952110 & 0.953542 & 0.953459 \\
24 & 0.954277 & 0.951891 & 0.953323 & 0.953240 \\
25 & 0.954006 & 0.951621 & 0.953052 & 0.952970 \\
26 & 0.953977 & 0.951592 & 0.953023 & 0.952941 \\
27 & 0.953969 & 0.951584 & 0.953015 & 0.952933 \\
28 & 0.999936 & 0.997436 & 0.998936 & 0.998849 \\
29 & 0.999733 & 0.997234 & 0.998733 & 0.998647 \\
30 & 0.999457 & 0.996958 & 0.998458 & 0.998371 \\
31 & 0.999411 & 0.996913 & 0.998412 & 0.998325 \\
32 & 0.999247 & 0.996749 & 0.998248 & 0.998161 \\
33 & 0.998896 & 0.996399 & 0.997897 & 0.997810 \\
34 & 0.998390 & 0.995894 & 0.997392 & 0.997305 \\
35 & 0.998130 & 0.995635 & 0.997132 & 0.997045 \\
\hline & & & &
\end{tabular}

TABle 6: Continued.

\begin{tabular}{lcccc}
\hline Node & CIM & PIDLF & SMM & Proposed method \\
\hline 36 & 0.999925 & 0.997425 & 0.998925 & 0.998838 \\
37 & 0.999544 & 0.997045 & 0.998544 & 0.998458 \\
38 & 0.999241 & 0.996743 & 0.998242 & 0.998155 \\
39 & 0.999161 & 0.996663 & 0.998162 & 0.998075 \\
40 & 0.999156 & 0.996658 & 0.998157 & 0.998070 \\
41 & 0.997629 & 0.995135 & 0.996631 & 0.996545 \\
42 & 0.997086 & 0.994593 & 0.996089 & 0.996002 \\
43 & 0.997026 & 0.994533 & 0.996029 & 0.995943 \\
44 & 0.997015 & 0.994523 & 0.996018 & 0.995932 \\
45 & 0.996936 & 0.994444 & 0.995939 & 0.995853 \\
46 & 0.996935 & 0.994443 & 0.995938 & 0.995852 \\
47 & 0.999890 & 0.997390 & 0.998890 & 0.998803 \\
48 & 0.999720 & 0.997221 & 0.998720 & 0.998634 \\
49 & 0.999327 & 0.996829 & 0.998328 & 0.998241 \\
50 & 0.999269 & 0.996771 & 0.998270 & 0.998183 \\
51 & 0.985447 & 0.982983 & 0.984462 & 0.984376 \\
52 & 0.985359 & 0.982896 & 0.984374 & 0.984288 \\
53 & 0.983837 & 0.981377 & 0.982853 & 0.982768 \\
54 & 0.982887 & 0.980430 & 0.981904 & 0.981819 \\
55 & 0.981600 & 0.979146 & 0.980618 & 0.980533 \\
56 & 0.980473 & 0.978022 & 0.979493 & 0.979408 \\
57 & 0.975240 & 0.972802 & 0.974265 & 0.974180 \\
58 & 0.974129 & 0.971694 & 0.973155 & 0.973071 \\
59 & 0.973792 & 0.971358 & 0.972818 & 0.972734 \\
60 & 0.973437 & 0.971003 & 0.972464 & 0.972379 \\
61 & 0.972846 & 0.970414 & 0.971873 & 0.971789 \\
62 & 0.972762 & 0.970330 & 0.971789 & 0.971705 \\
63 & 0.972679 & 0.970247 & 0.971706 & 0.971622 \\
64 & 0.972407 & 0.969976 & 0.971435 & 0.971351 \\
65 & 0.972207 & 0.969777 & 0.971235 & 0.971151 \\
66 & 0.974214 & 0.971779 & 0.973240 & 0.973156 \\
67 & 0.974214 & 0.971779 & 0.973240 & 0.973156 \\
68 & 0.962995 & 0.960588 & 0.962032 & 0.961949 \\
69 & 0.962993 & 0.960586 & 0.962030 & 0.961947 \\
\hline & & & &
\end{tabular}


TABle 7: Comparison of the performance of the proposed approach.

\begin{tabular}{lcccc}
\hline & \multicolumn{4}{c}{ Number of iterations } \\
& CIM & PIDLF & SMM & Proposed method \\
\hline IEEE 15 bus & 4 & 4 & 4 & 4 \\
IEEE 69 bus & 5 & 7 & 4 & 4 \\
\hline
\end{tabular}

\section{Conclusion}

In this new approach, a load flow program based on the backward/forward sweep concept is used to solve load flow problems in radial distribution systems, with an improvement in the element ordering that does not require the use of any complex numbering of branches or the use of any matrix calculation. Simply by devising the system element into a main line and its derivations, the flow calculation is reduced due to the use of only algebraic expressions without any trigonometric functions or matrix calculation and by ensuring minimum number of searching for connections between nodes.

The proposed load flow method is easy to implement in the current Moroccan medium-voltage system, load data can be obtained and updated by the advanced metering infrastructure (AMI), already existing and used by consumers connected to the medium-voltage distribution network in Morocco. The needed data of other loads can be obtained by equipping MV/LV substation by using digital meters and connecting them to the advanced metering infrastructure network. The line data can be established by collecting the impedance of all the branches constituting the network, and any change in the topology structure can be detected manually or by the existing SCADA, installed already in HV/ MV substation in Morocco, and used as medium-voltage network remote control system.

The proposed method can be exploited in several distribution network applications: voltage control, demandside management, and energy demand management.

Limitation of the proposed method is that it can be used only for the radial distribution system and not for meshed distribution systems or transmission systems.

\section{Data Availability}

The IEEE 15-bus structure, line date, and load data used to support the simulation of the proposed load flow method are included within the article. The result data of the other existing load flow methods, including Primitive Impedance based Distribution Load Flow (PIDLF), Current Injections based Distribution Load Flow (CIM), and Fast Decoupled Single Matrix Model Distribution Load Flow (SMM), used to support the validate the result obtained from the proposed method are included within the article. The Matlab code developed and used to perform the proposed method is available from the corresponding author upon request.

\section{Conflicts of Interest}

The authors declare that they have no conflicts of interest.

\section{References}

[1] L. K. Kiarie, P. K. Langat, and C. M. Muriithi, "Application of spritz encryption in smart meters to protect consumer data," Journal of Computer Networks and Communications, vol. 2019, Article ID 5910528, 10 pages, 2019.

[2] A. Kailas, V. Cecchi, and A. Mukherjee, "A survey of communications and networking technologies for energy management in buildings and home automation," Journal of Computer Networks and Communications, vol. 2012, Article ID 932181, 12 pages, 2012.

[3] K. Gajowniczek and T. Zabkowski, "Simulation study on clustering approaches for short-term electricity forecasting," Complexity, vol. 2018, Article ID 3683969, 21 pages, 2018.

[4] R. H. Inman, H. T. C. Pedro, and C. F. M. Coimbra, "Solar forecasting methods for renewable energy integration," Progress in Energy and Combustion Science, vol. 39, no. 6, pp. 535-576, 2013.

[5] Y. Yang, "Practical robust optimization method for unit commitment of a system with integrated wind resource," Mathematical Problems in Engineering, vol. 2017, Article ID 5208290, 13 pages, 2017.

[6] P. Yi, A. Iwayemi, and C. Zhou, "Building automation networks for smart grids," International Journal of Digital Multimedia Broadcasting, vol. 2011, Article ID 926363, 12 pages, 2011

[7] M. Venkata Kirthiga and S. Arul Daniel, "Computational techniques for autonomous microgrid load flow analysis," ISRN Power Engineering, vol. 2014, Article ID 742171, 12 pages, 2014.

[8] H. Wu, Q. Zhai, X. Guan, F. Gao, and H. Ye, "Securityconstrained unit commitment based on a realizable energy delivery formulation," Mathematical Problems in Engineering, vol. 2012, Article ID 178193, 22 pages, 2012.

[9] S. Kolen, S. Dähling, T. Isermann, and A. Monti, "Enabling the analysis of emergent behavior in future electrical distribution systems using agent-based modeling and simulation," Complexity, vol. 2018, Article ID 3469325, 16 pages, 2018.

[10] B. Wang, X. Hu, P. Shen, W. Ji, Y. Cao, and J. Tang, "A flexible load control strategy for distribution network to reduce the line losses and to eliminate the transmission congestion," Mathematical Problems in Engineering, vol. 2017, Article ID 6343025, 16 pages, 2017.

[11] H. E. Espitia, I. Machón-González, H. López-García, and G. Díaz, "Proposal of an adaptive neurofuzzy system to control flow power in distributed generation systems," Complexity, vol. 2019, Article ID 1610898, 16 pages, 2019.

[12] N. I. Voropai and B. Bat-Undraal, "Multicriteria reconfiguration of distribution network with distributed generation," Journal of Electrical and Computer Engineering, vol. 2012, Article ID 317315, 8 pages, 2012.

[13] N. H. Moin and T. Yuliana, "Three-phase methodology incorporating scatter search for integrated production, inventory, and distribution routing problem," Mathematical Problems in Engineering, vol. 2015, Article ID 304981, 11 pages, 2015.

[14] S. A. Taher and S. A. Afsari, "Optimal location and sizing of UPQC in distribution networks using differential evolution algorithm," Mathematical Problems in Engineering, vol. 2012, Article ID 838629, 20 pages, 2012.

[15] T. Erdelić and T. Carić, "A survey on the electric vehicle routing problem: variants and solution approaches," Journal of Advanced Transportation, vol. 2019, Article ID 5075671, 48 pages, 2019. 
[16] W. F. Tinney and C. E. Hart, "Power flow solution by Newton's method," IEEE Transactions on Power Apparatus and Systems, vol. PAS-86, no. 11, pp. 1449-1460, 1967.

[17] R. G. Wasley and M. A. Shlash, "Newton-Raphson algorithm for 3-phase load flow," Proceedings of the Institution of Electrical Engineers, vol. 121, no. 7, pp. 630-638, 1974.

[18] J.-J. Deng and H.-D. Chiang, "Convergence region of Newton iterative power flow method: numerical studies," Journal of Applied Mathematics, vol. 2013, Article ID 509496, 12 pages, 2013.

[19] D. Thukaram, H. M. Wijekoon Banda, and J. Jerome, "A robust three phase power flow algorithm for radial distribution systems," Electric Power Systems Research, vol. 50, no. 3, pp. 227-236, 1999.

[20] F. Zhang and C. S. Cheng, "A modified Newton method for radial distribution system power flow analysis," IEEE Transactions on Power Systems, vol. 12, no. 1, 1997.

[21] J. L. Guardado, F. Rivas-Davalos, J. Torres, S. Maximov, and E. Melgoza, "An encoding technique for multiobjective evolutionary algorithms applied to power distribution system reconfiguration," The Scientific World Journal, vol. 2014, Article ID 506769, 9 pages, 2014.

[22] S. K. Goswami and S. K. Basu, "A new algorithm for the reconfiguration of distribution feeders for loss minimization," IEEE Transactions on Power Delivery, vol. 7, no. 3, pp. 1484-1491, 1992.

[23] A. Dukpa, B. Venkatesh, and M. El-Hawary, "Application of continuation power flow method in radial distribution systems," Electric Power Systems Research, vol. 79, no. 11, pp. 1503-1510, 2009.

[24] J. A. Michline Rupa and S. Ganesh, "power flow analysis for radial distribution system using backward/forward sweep method," World Academy of Science, Engineering and Technology International Journal of Electrical and Computer Engineering, vol. 8, no. 10, 2014.

[25] A. AppaRao and M. Win Babu, "Forward sweeping method for solving radial distribution networks," International Journal of Advanced Research in Electrical, Electronics and Instrumentation Engineering, vol. 2, no. 9, September 2013.

[26] T. H. Chen, M. S. Chen, K. J. Hwang, P. Kotas, and E. A. Chebli, "Distribution system power flow analysis-a rigid approach," IEEE Transactions on Power Delivery, vol. 6, no. 3, pp. 1146-1152, 1991.

[27] K. Nagaraju, S. Sivanagaraju, T. Ramana, and P. V. Prasad, “A novel load flow method for radial distribution systems," International Journal of Power and Energy Systems, vol. 31, no. 1, 2011.

[28] D. Shirmohammadi, H. W. Hong, A. Semlyen, and G. X. Luo, "A compensation-based power flow method for weakly meshed distribution and transmission networks," IEEE Transactions on Power Systems, vol. 3, no. 2, pp. 753-762, 1988.

[29] E. Bompard, E. Carpaneto, G. Chicco, and R. Napoli, "Convergence of the backward/forward sweep method for the load-flow analysis of radial distribution systems," International Journal of Electrical Power \& Energy Systems, vol. 22, no. 7, pp. 521-530, 2000.

[30] H. D. Chiang and M. E. Baran, "On the existence and uniqueness of load flow solution for radial distribution power network," IEEE Transactions on Circuits and Systems, vol. 37, no. 3, pp. 410-416, 1990.

[31] M. Baran and F. Wu, "Network reconfiguration in distribution systems for loss reduction and load balancing," IEEE Transactions on Power Delivery, vol. 4, no. 3, 1989.
[32] T. Thakur and J. Dhiman, "A new approach to load flow solutions for radial distribution system," in Proceedings of the 2006 IEEE/PES Transmission \& Distribution Conference and Exposition: Latin America, Caracas, Venezuela, August 2006.

[33] R. D. Zimmerman and H. D. Hsiao-Dong Chiang, "Fast decoupled power flow for unbalanced radial distribution systems," IEEE Transactions on Power Systems, vol. 10, no. 4, pp. 2045-2052, 1995.

[34] T. Sathiyanarayanan and M. Sydulu, "Load flow analysis for radial and mesh connected distribution systems," International Journal of Science and Research (IJSR), vol. 3, no. 6, 2014.

[35] S. Ouali and A. Cherkaoui, "Load Flow Analysis For Moroccan Medium Voltage Distribution System," in Proceedings of Engineering and Technology-PET Conférence Internationale en Automatique \& Traitement de Signal (ATS2018), vol. 36, pp. 10-16, Tanger, Maroc, March 2018. 


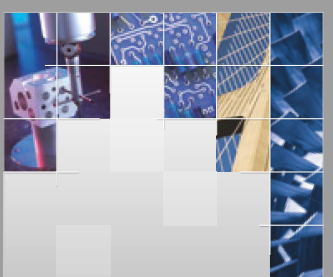

\section{Enfincering}
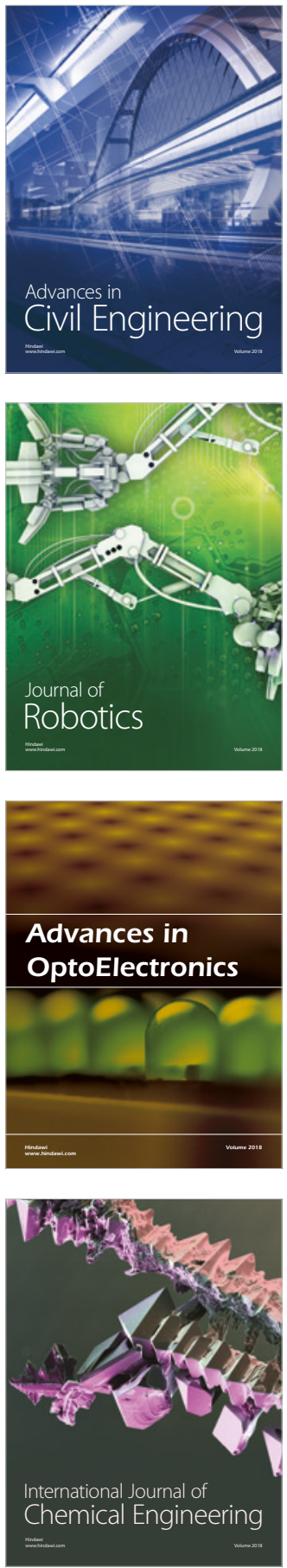

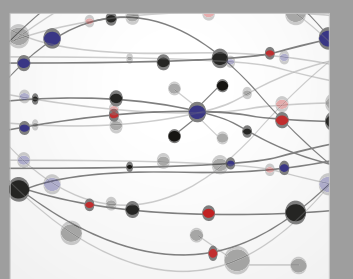

\section{Rotating \\ Machinery}

The Scientific World Journal

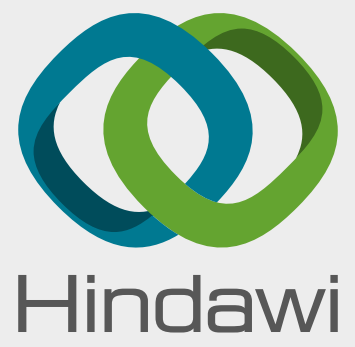

Submit your manuscripts at

www.hindawi.com
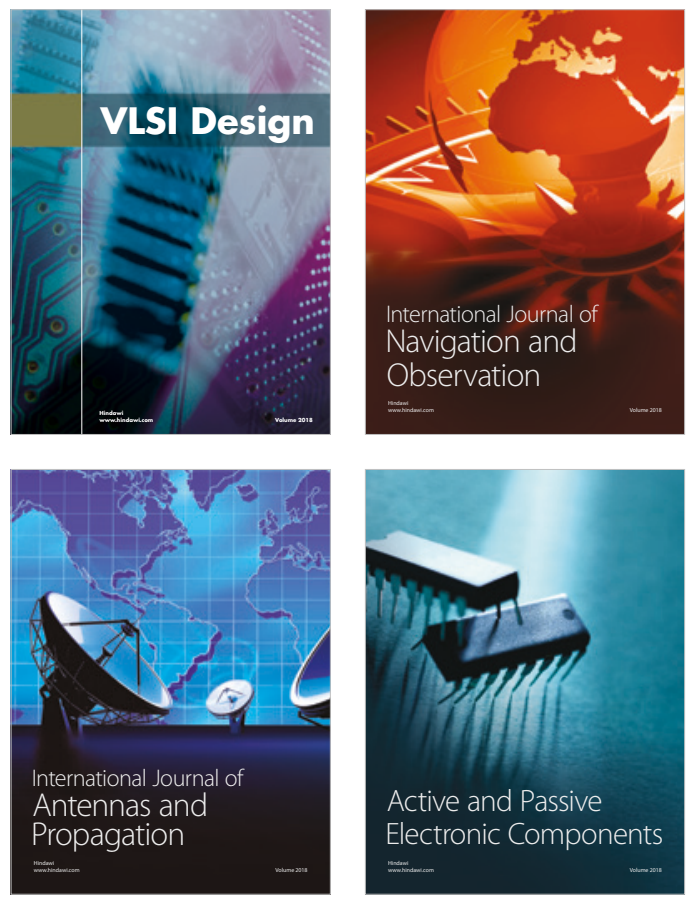
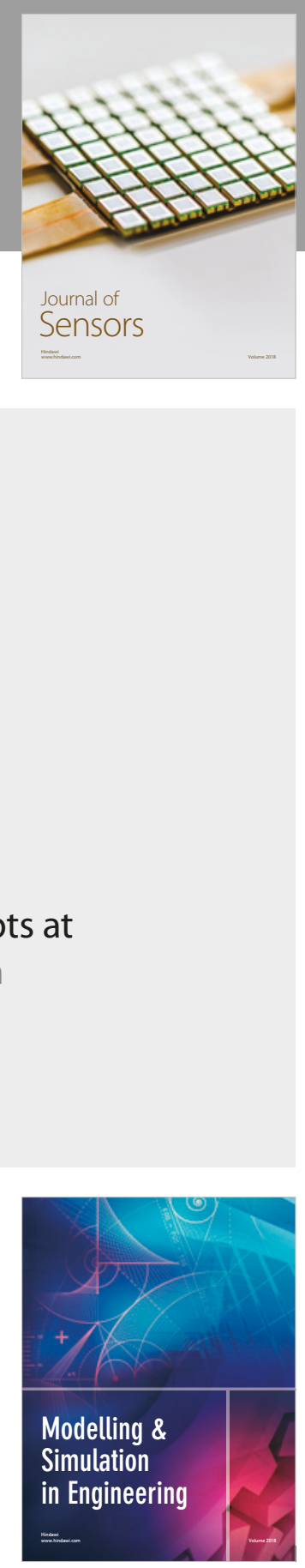

\section{Advances \\ Multimedia}
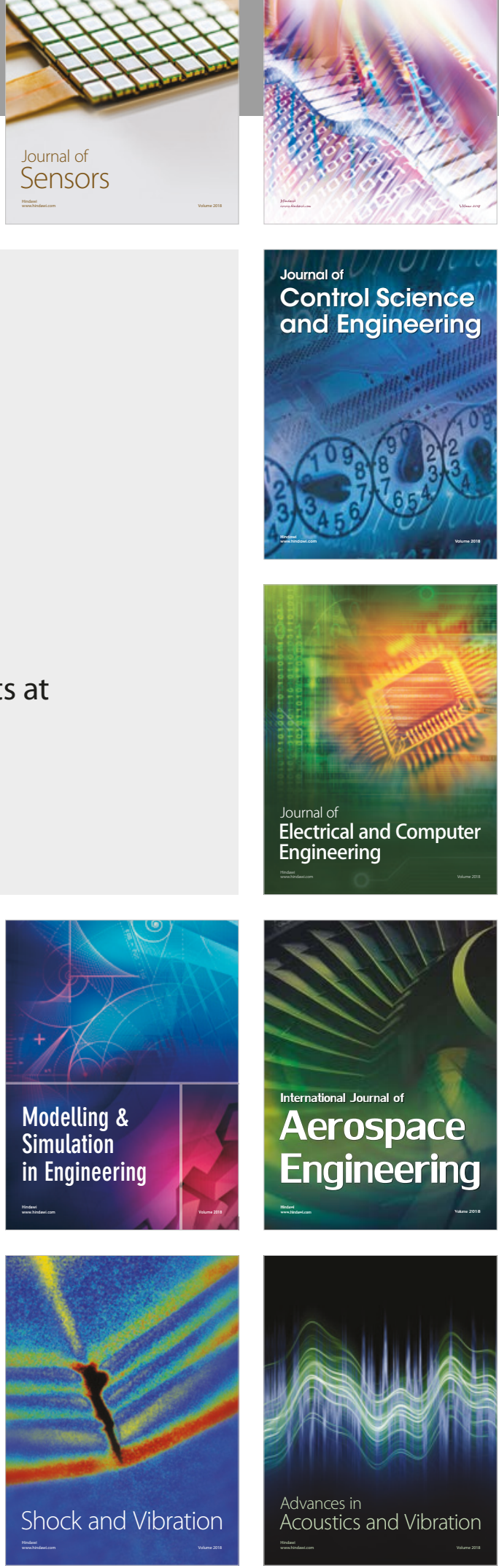